\title{
Impact of size polydispersity on the nature of Lennard-Jones liquids
}

\author{
Trond S. Ingebrigtsen ${ }^{1, *}$ and Hajime Tanaka ${ }^{1, \dagger}$ \\ ${ }^{1}$ Institute of Industrial Science, University of Tokyo, \\ 4-6-1 Komaba, Meguro-ku, Tokyo 153-8505, Japan
}

(Dated: August 24, 2018)

\begin{abstract}
Polydisperse fluids are encountered everywhere in biological and industrial processes. These fluids naturally show a rich phenomenology exhibiting fractionation and shifts in critical point and freezing temperatures. Here, we study the impact of size polydispersity on the basic nature of Lennard-Jones (LJ) liquids, which represent most molecular liquids without hydrogen bonds, via two- and threedimensional molecular dynamics computer simulations. A single-component liquid constituting spherical particles and interacting via the LJ potential is known to exhibit strong correlations between virial and potential energy equilibrium fluctuations at constant volume. This correlation significantly simplifies the physical description of the liquid, and these liquids are now known as Roskilde-simple (RS) liquids. We show that this simple nature of the single-component LJ liquid is preserved even for very high polydispersities (above $40 \%$ polydispersity for the studied uniform distribution). We also investigate isomorphs of moderately polydisperse LJ liquids. Isomorphs are curves in the phase diagram of RS liquids along which structure, dynamics, and some thermodynamic quantities are invariant in dimensionless units. We find that isomorphs are a good approximation even for polydisperse LJ liquids. The theory of isomorphs thus extends readily to multi-component systems and can be used to improve even further the understanding of these intriguing systems.
\end{abstract}

\section{INTRODUCTION}

Polydisperse fluids are ubiquitous in both biological and industrial processes 1.2 . Examples of polydisperse systems are: plastic materials, liquid foams, asphalt, micelles, and more. The property that typically varies among the constituent molecules is the charge, mass, and size. The fluids' dispersity naturally induces a rich phenomenology not encountered in their singlecomponent counterparts. As a consequence, polydisperse fluids have been the focus of a number of studies both theoretically $\underline{3}^{\underline{3}-11}$ and via computer simulations ${ }^{11}-\underline{31}$, as well as in experiments ${ }^{32}-40$.

These investigations revealed that polydisperse fluids exhibit a very rich phase diagram with shifts in critical point and freezing temperatures, as well as many-solid/fluid coexistence regions 10,41 . The coexisting phases in fact show fractionation, i.e., the dispersed variable displays a distribution different from the parent in each phase. Moreover, polydisperse fluids exhibit terminal polydispersity beyond which the solid is not stable $10,25,31$. Polydispersity is thus regarded as an important factor to improve the glass-forming ability of liquids and to control the liquid fragility $22,23,36,42-44$.

Predicting the behavior of polydisperse fluids is a complex task, and several theoretical approaches have emerged to face the challenges of polydisperse fluids (see, e.g., Refs. 8, 9, 11, and 26). The complex nature of the problem is enhanced by the fact that quasi-universal relations $\underline{45}-\underline{47}$ for monatomic fluids, possessing great predictive power, break down in increasingly polydisperse fluids 31.48 . One such example is Rosenfeld's excess entropy scaling ${ }^{45,46}$ in which a dimensionless transport coefficient is correlated to the excess entropy with respect to an ideal gas. The latter observation for polydisperse flu- ids stands in contrast to confined fluids where the quasiuniversal relations are very robust as the degree of confinement is increased $\underline{49}-53$.

Explanations for quasi-universal relations in monatomic fluids were recently presented from a constant-potential-energy $N V U$-dynamics perspective $e^{54}-57$, as well as from an exponential pair potential perspective ${ }^{58,59}$. In these perspectives, the quasi-universal relations are attributed to the monatomic fluids being so-called Roskilde-simple $\underline{\underline{60}} \underline{\underline{66}}$.

Roskilde-simple (RS) liquids have been detailed in a number of papers ${ }^{60-64}$ and appear to be simpler than other types of liquids 66 . RS liquids are characterized by having strong correlations between virial and potential energy equilibrium fluctuations at constant volume. In simulations of model liquids, the single-component Lennard-Jones (SCLJ) liquid, among many others, has been identified to belong to the class of RS liquids. Some experimental liquids have also been identified as $\mathrm{RS}^{67} \underline{\underline{69}}$. More specifically, the class of RS liquids is believed to include most or all van der Waals and metallic liquids, but to exclude most or all covalent-bonding, hydrogenbonding, strongly ionic, and dipolar liquids 60 . Moreover, RS liquids are characterized by having isomorphs. Isomorphs are curves in the phase diagram of RS liquids along which structure, dynamics, and some thermodynamic quantities are invariant in dimensionless units; a property that is not limited to the stable liquid region but also valid in the supercooled liquid and even out-ofequilibrium $\underline{63,70}$.

Recently, Bagchi and coworkers 25.31 studied Lindemann's melting and Hansen-Verlet's freezing criterion in size polydisperse LJ liquids. The authors showed surprisingly that terminal polydispersity can be analyzed in terms of these rules. It was also shown that order- 
parameter maps for these liquids are not independent but highly correlated. We note that melting and freezing lines of RS liquids are isomorphs from which the rules of Lindemann and Hansen-Verlet follow $\underline{63}$. In fact, correlated order-parameter maps also follow from the existence of isomorphs in the phase diagram $\underline{63}$.

The observations of Bagchi and coworkers imply that polydisperse LJ liquids retain the character of RS liquids. Conversely, the breakdown of quasi-universal relations in polydisperse fluids may imply that polydisperse LJ liquids do not remain RS. Thus, we investigate in the following the effect of size polydispersity on strong virialpotential energy correlation in a systematic way to shed light on melting and freezing rules and quasi-universal behavior in polydisperse fluids. We note that previous investigations of multi-component RS liquids focused only on binary mixtures ${ }^{60,64,66}$.

Section 1 describes the applied methods and simulated model systems. Section [II introduces RS liquids and explain their characteristics. In Sec. IV we show results from simulating size polydisperse LJ liquids in both two and three dimensions, in particular, the effect of size polydispersity on strong virial-potential energy correlation. Section $\nabla$ discusses the physics behind our observations. Finally, Sec. VI] summarizes our work and presents an outlook.

\section{SIMULATION AND MODEL DETAILS}

We apply NVT molecular dynamics computer simulations $\underline{71}-\underline{73}$ to study size polydisperse LJ liquids in two and three dimensions. The RUMD package (see http://rumd.org) is used for three-dimensional simulations while an in-house code is used for two-dimensional simulations. Pros and cons against applying constantparticle-number simulations to study polydisperse fluids are discussed in Ref. 74.

The polydisperse LJ liquid provides a convenient framework for initiating theoretical investigations of polydispersity 18,31 , but has also been applied to mimic the behavior of for instance hydrocarbon or polymer solutions, idealized models for spherical micelles in water above the critical micelle concentration, and polystyrene lattices stabilized with polyoxyethylene chains $\underline{41}$.

If the total potential energy of the system is given by $U=\sum_{i<j} v\left(r_{i j}\right)$, the size polydisperse LJ pair potential is

$$
v\left(r_{i j}\right)=4 \epsilon\left[\left(\frac{\sigma_{i j}}{r_{i j}}\right)^{12}-\left(\frac{\sigma_{i j}}{r_{i j}}\right)^{6}\right]
$$

where $\sigma_{i j}$ and $\epsilon$ set, respectively, the length and energy scale of the pair interaction between particle $i$ and particle $j(i, j=1, \ldots, N$, in which $N$ is the number of particles). As the study is focused on size polydisperse LJ liquids: $\epsilon$ is independent of the pair interaction and fixed in simulations. The parameter $\sigma_{i j}$ is defined from the
Lorentz-Berthelot mixing rule $\frac{75}{\underline{5}}$ given by

$$
\sigma_{i j}=\left(\sigma_{i}+\sigma_{j}\right) / 2 .
$$

In this study, $\sigma_{i}$ and $\sigma_{j}$ are evenly distributed on an interval from $\sigma^{\min }$ to $\sigma^{\max }$. The polydispersity of the liquid $\delta$ is defined by

$$
\delta \equiv \sigma^{S T D}\left(\sigma_{i}\right) / \bar{\sigma}_{i}=\frac{1}{\sqrt{3}} \frac{\sigma^{\max }-\sigma^{\min }}{\sigma^{\max }+\sigma^{\min }},
$$

where $\sigma^{S T D}\left(\sigma_{i}\right)$ and $\bar{\sigma}_{i}$ are, respectively, the dispersed variable's standard deviation and mean. We study polydispersities in the range $\delta=0 \%$ to $\delta=52 \%$ and apply a truncated-and-shifted pair potential cutoff at $r_{c}=2.5 \sigma_{i j}$. We note that care should be taken when comparing the absolute polydispersity number $\delta$ amongst different distributions 18 .

The units of the simulation are defined by setting the mean values over the $N$ particles to unity, i.e., $\sigma_{\bar{N} \bar{N}}=1$, $\epsilon_{\bar{N} \bar{N}}=1, m_{\bar{N}}=1$. All particle masses are identical (and equal to unity). Figure 1 shows pair potentials of Eq. (11) with $\sigma_{i j}=0.1,1.0,1.9$ and $\epsilon=1$. The latter corresponds to the range of LJ potentials used in simulations of a size polydisperse LJ liquid with $\delta=$ $52 \%$ (i.e., $\sigma^{\min }=0.1$ and $\sigma^{\max }=1.9$ ). For this high polydispersity the simulated pair potentials cover a very broad range of potentials.

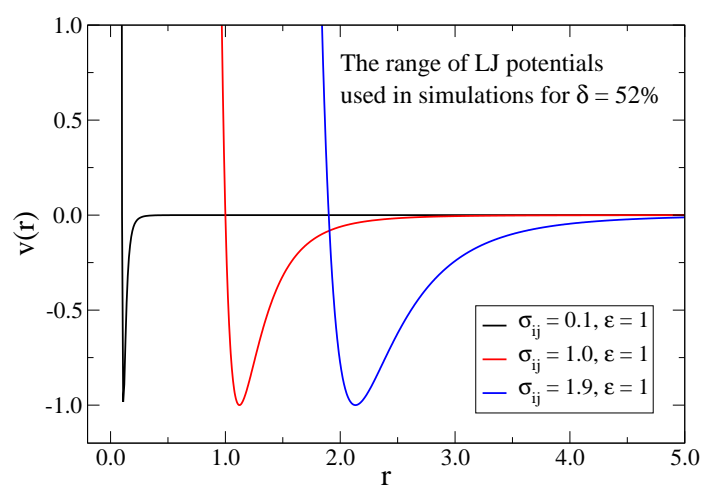

FIG. 1: The range of LJ potentials used in simulations of a polydisperse LJ liquid with $\delta=52 \%$.

The two-dimensional simulations use $N=1000$ particles, whereas the three-dimensional simulations use $N=$ 5000 particles. The finite-size effects are larger in two dimensions than in three dimensions. In either case, however, finite-size effects are observed to be small for the quantities studied in this paper and no additional averaging is performed. 


\section{ROSKILDE-SIMPLE LIQUIDS}

RS liquids and their associated simple properties have been detailed in a number of papers, starting with a series of five papers ${ }^{60}-64$. RS liquids are characterized by having strong correlations between virial $W$ and potential energy $U$ equilibrium fluctuations at constant volume. The virial-potential energy correlation is quantified via Pearson's correlation coefficient $R$ given by

$$
R=\frac{\langle\Delta W \Delta U\rangle}{\sqrt{\left\langle(\Delta W)^{2}\right\rangle} \sqrt{\left\langle(\Delta U)^{2}\right\rangle}}
$$

in which $\Delta$ is deviation from mean values, $\langle\ldots\rangle$ denotes $N V T$ ensemble averages, and $-1 \leq R \leq 1$. RS liquids are defined pragmatically by requiring $R \geq 0.90$. $R$ depends on the state point investigated but has been shown to be high in large parts of the phase diagram or not at all.

Only inverse power-law (IPL) fluids with $r^{-n}$ pair potentials are perfectly correlating $(R=1$ since $\Delta W=$ $(n / 3) \Delta U)$, but many models $60,66,76$, such as the SCLJ liquid, the Kob-Andersen binary LJ mixture, the LewisWahnström OTP model, the asymmetric dumbbell model, identical chain length LJ-bead polymer models, and some experimental liquids ${ }^{67-69}$, have been shown to belong to the class of RS liquids.

The origin behind strong correlation in the SCLJ liquid is as follows. The LJ pair potential can be approximated by an IPL potential in the repulsive part $\stackrel{77-79}{ }$, i.e., below the potential mininum with an IPL-exponent $n \approx 18$. The required exponent is larger than the repulsive term $r^{-12}$ encountered in the LJ potential as the effect of attraction is to increase the steepness of the repulsive part. However, contributions to the fluctuations come from pair distances around the pair potential minimum 61,66 . As a consequence, one must add a linear term and a constant to the IPL potential to obtain a proper description of the LJ potential:

$$
v_{L J}(r) \approx A r^{-n}+B+C r .
$$

The constraint of constant volume in the NVT ensemble has the following effect: When one nearest-neighbor distance increases another one decreases. Thus, upon summation the contribution from the linear term to $U$ and $W$ is almost constant and vanishes with respect to the fluctuations. For a very recent review of RS liquids, see Ref. 80.

As mentioned, RS liquids are associated with a number of simple properties. RS liquids are, for instance, characterized by having isomorphs to a good approximation 63 . Consider two state points in a liquid's phase diagram with density and temperature $\left(\rho_{1}, T_{1}\right)$ and $\left(\rho_{2}, T_{2}\right)$. These two state points are defined to be isomorphic if the following holds: Whenever a microconfiguration of state point (1) and of state point (2) have the same reduced coordinates, $\rho_{1}^{1 / 3} \mathbf{R}^{(1)}=\rho_{2}^{1 / 3} \mathbf{R}^{(2)}(\mathbf{R}$ denotes a $3 N$ - dimensional configurational-space vector and $\rho \equiv N / V$, in which $V$ is the volume), these two configurations have proportional Boltzmann factors, i.e.,

$$
e^{-U\left(\mathbf{R}^{(1)}\right) / k_{B} T_{1}}=C_{12} e^{-U\left(\mathbf{R}^{(2)}\right) / k_{B} T_{2}} .
$$

Here $C_{12}$ is a constant and depends only on the state points (1) and (2). An isomorph is defined as a continuous curve of state points that are all pairwise isomorphic. For IPL fluids $C_{12}=1$, and isomorphs thus encapsulate the well-known scaling properties 81 of these systems. However, for all other RS liquids $C_{12} \neq 1$; accordingly, the state points can be represented effectively on a onedimensional phase diagram only for certain properties 63 .

An immediate consequence of isomorphs is that a number of quantities are invariant along these curves. The invariants include the structure and dynamics in reduced units and some thermodynamic quantities, e.g., the excess entropy with respect to an ideal gas $s_{e x} \equiv S_{e x} / N$. We note that reduced units refer to macroscopic quantities, such as $\rho^{-1 / 3}$ and $T$, rather than the usual approach in simulations via microscopic quantities (see Ref. 63 for details).

For RS liquids, temperature separates 65 in a function of density and of excess entropy: $k_{B} T=h(\rho) f\left(s_{\text {ex }}\right)$. From this equation, one observes the isomorph invariant $h(\rho) / T$ which was used to define the so-called isomorph scaling 65,82 , in which the reduced relaxation time $\tilde{\tau}_{\alpha}$ is a function $\tilde{\tau}_{\alpha}=f(h(\rho) / T)$. Isomorph scaling explains classical density scaling ${ }^{83}$ for which $\tilde{\tau}_{\alpha}=f\left(\rho^{\gamma} / T\right)$, where $\gamma$ is a fitting exponent. Density scaling is valid only for relatively small density variations, whereas isomorph scaling works even when the density variation is large ${ }^{82}$. Additionally, the isomorph invariant $h(\rho) / T$ provides a convenient way to generate isomorphs since $h(\rho)$ inherits the analytical structure of the potential energy function 65 .

There exist additional simple observations for RS liquids than mentioned so far, e.g., the dominance of firstcoordination shell interactions 66,84 , extension to nontrivial boundary conditions such as nanoconfinement ${ }^{52}$ or shear-flow $\stackrel{70}{ }$, crystalline isomorphs ${ }^{85}$, and the relevance of Rosenfeld-Tarazona's expressions ${ }^{86}$ for these liquids 87 . The interested reader is referred to these and other papers for more information.

Finally, a reformulation of isomorphs was very recently presented $^{88}$. If $\mathbf{R}_{a}$ and $\mathbf{R}_{b}$ denote two different $3 N$-dimensional configurational-space vectors of a chosen state point, the isomorphic condition is

$$
U\left(\mathbf{R}_{a}\right)<U\left(\mathbf{R}_{b}\right) \Rightarrow U\left(\lambda \mathbf{R}_{a}\right)<U\left(\lambda \mathbf{R}_{b}\right),
$$

where $\lambda$ is a scalar. This definition encapsulates the previous definition of isomorphs as a (very good) first-order approximation ${ }^{88}$. The mentioned invariants are exact within this reformulation, too. We note, however, that the constant volume heat capacity is not formally invariant. 


\section{RESULTS FROM POLYDISPERSE LJ LIQUIDS}

The simulations in this paper were performed both in two and three dimensions, and thus we discuss each case separately beginning with the two-dimensional results.

\section{A. Simulations in two dimensions}

The investigation is initiated by considering the effect of size polydispersity on the structure and dynamics of the SCLJ liquid in two dimensions at $\rho=0.75$ and $T=$ 0.70 . For $0 \%$ polydispersity, this state point corresponds to the liquid phase.

Figure 2(a) presents radial distribution functions (RDFs) upon the increase of polydispersity from $\delta=0 \%$ to $\delta=52 \%$, whereas Fig. 2(b) shows bond-orientationalorder functions 22,89 (BOFs) defined via

$$
g_{6}(r) \equiv \frac{L^{2}}{2 \pi r \Delta r N(N-1)} \sum_{i \neq k} \delta\left(r-\left|\mathbf{r}_{i k}\right|\right) \psi_{6}^{i} \psi_{6}^{k *}
$$

The division with $g(r)$ in Fig. 2(b) approximately accounts for effects due to positional order in the liquid ${ }^{22,90}$. In the equation above, $\delta$ denotes Dirac's $\delta$-function, $\mathbf{r}_{i k} \equiv$ $\mathbf{r}_{i}-\mathbf{r}_{k}, L$ is the box length, and $\psi_{6}^{i} \equiv 1 / n_{i} \sum_{m=1}^{n_{i}} e^{j 6 \theta_{m}^{i}}$, in which $n_{i}$ is the number of nearest neighbors of particle $i$, and $\theta_{m}^{i}$ is the angle between the vector $\mathbf{r}_{m i}$ and the $x$-axis (particle $m$ is a neighbor of particle $i$ ). Nearest-neighbor particles are identified via a Voronoiconstruction 75 . Finally, Fig. 2(c) shows mean-square displacements (MSDs).

Both measures of structural order (Figs. 2(a) and (b)) are seen to vanish as polydispersity is increased from $\delta=0 \%$ to $\delta=52 \%$ at constant density and temperature. The MSD shows approximately one order-of-magnitude decrease in the diffusion coefficient over the same variation in polydispersity. Introducing size polydispersity has thus a pronounced effect on the SCLJ liquid. The latter results confirm several previous simulations of polydisperse hard-sphere and LJ liquids $12,18,25$. We note that if the volume (area) fraction $\phi \equiv 1 / L^{2} \sum_{i} \pi\left(\sigma_{i} / 2\right)^{2}$ of the system is kept constant, instead of density, a similar diminishment of structural correlations is observed. However, in the case of the dynamics an enhancement of the mobility is seen 23 .
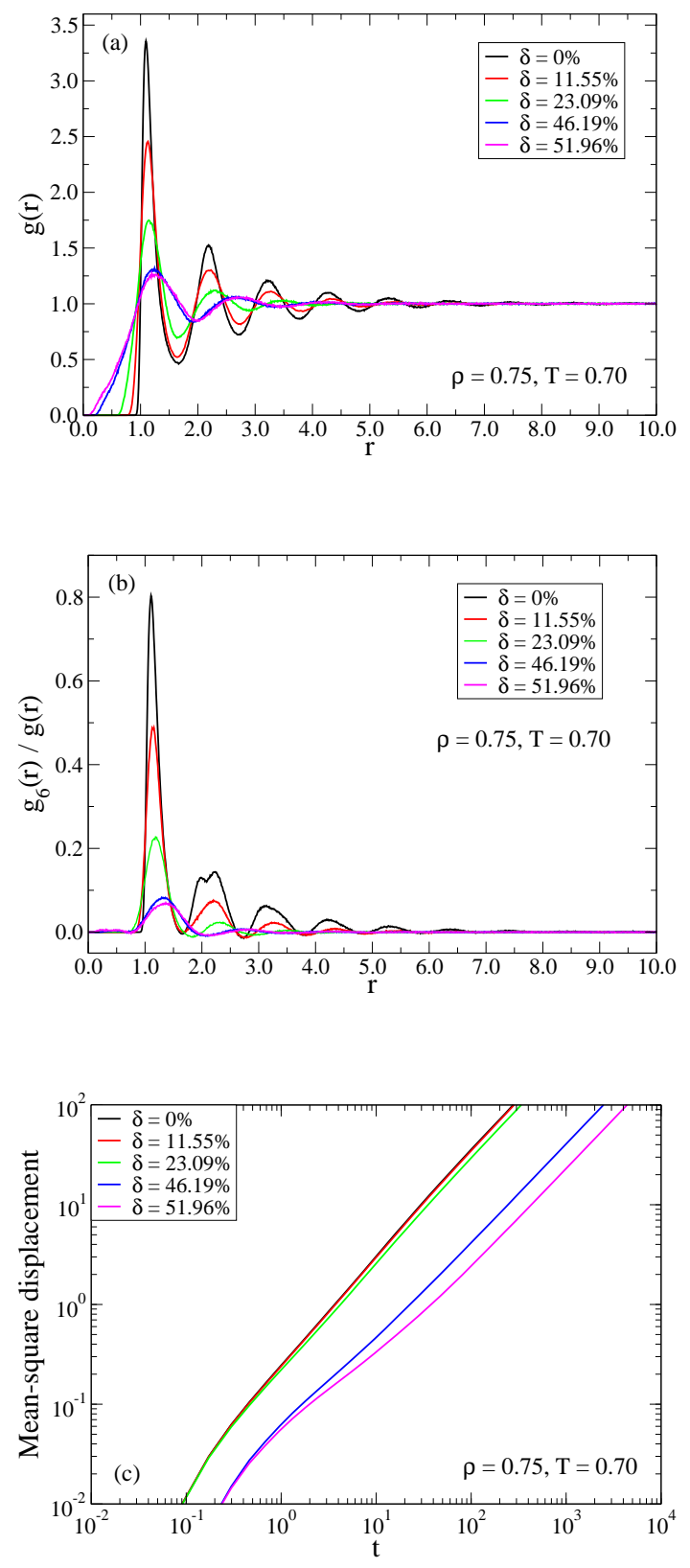

FIG. 2: Structural and dynamical effects of size polydispersity on the SCLJ liquid in two dimensions at $\rho=0.75$ and $T=$ 0.70. (a) Radial distribution functions (RDFs). (b) Bondorientational-order functions (BOFs, see Eq. (8)). (c) Meansquare displacements (MSDs). Size polydispersity is seen to diminish structural order and slow down dynamics at constant density and temperature.

Furthermore, Fig. 3 shows a snapshot of a particle configuration for the size polydisperse LJ liquid with $\rho$ $=0.75, T=0.70, \delta=52 \%$. Each particle is represented according to its value of $\sigma_{i}$. A multitude of different sizes are present at this high polydispersity exemplifying again the rich and non-trivial diversity of the liquids studied. 


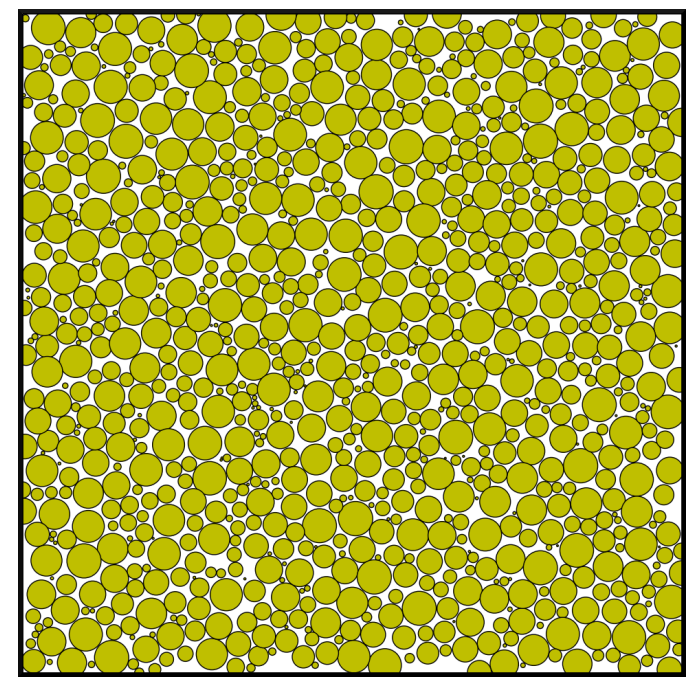

FIG. 3: A snapshot of a particle configuration $(N=1000)$ for a highly size polydisperse LJ liquid with $\rho=0.75, T=0.70$, $\delta=52 \%$. Each particle is represented according to its value of $\sigma_{i}$ : As the LJ potential is continuous some of the disks overlap slightly. A multitude of different sizes are present at this high polydispersity.

The study is now turned towards the assessment of the effect of size polydispersity from the viewpoint of $\mathrm{RS}$ liquids. In this connection, we note that previous simulations of RS liquids were performed only in three dimensions. The simulations in this section are twodimensional.

Figure 4 shows the correlation coefficient $R$ as function of polydispersity $\delta$ at both constant density $\rho$ (black data points) and constant volume fraction $\phi$ (red data points). For the three-dimensional SCLJ liquid, a typical value for the correlation coefficient is $R \approx 0.95$. The inset shows the density-scaling exponent ${ }^{63,65} \gamma \equiv$ $\langle\Delta U \Delta W\rangle /\left\langle(\Delta U)^{2}\right\rangle$ as function of polydispersity (see Sec. III).

At constant density, we observe both a decrease and an increase in $R$ depending on the starting state point at $0 \%$ polydispersity. This observation is expected as the phase diagram for polydisperse liquids shows very rich phenomenology ${ }^{10,41}$. Although a decrease in $R$ is observed for the two highest densities $(\rho=0.95$ and $\rho=$ $0.85)$ all state points investigated are RS. For the lowest density $(\rho=0.75)$ the liquid becomes RS at high polydispersities. In fact, the results show that polydisperse LJ liquids at $52 \%$ polydispersity are RS liquids. This observation is non-trivial given the complex nature of polydisperse liquids (see also Fig. 3).

In the case of constant volume fraction, however, we observe a significant decrease in $R$ as function of polydispersity. An increase in polydispersity, at constant volume fraction, is accompanied by a decrease in pressure. This is in contrast to constant density which increases the pressure; Table $\llbracket$ shows selected polydispersities and cor-

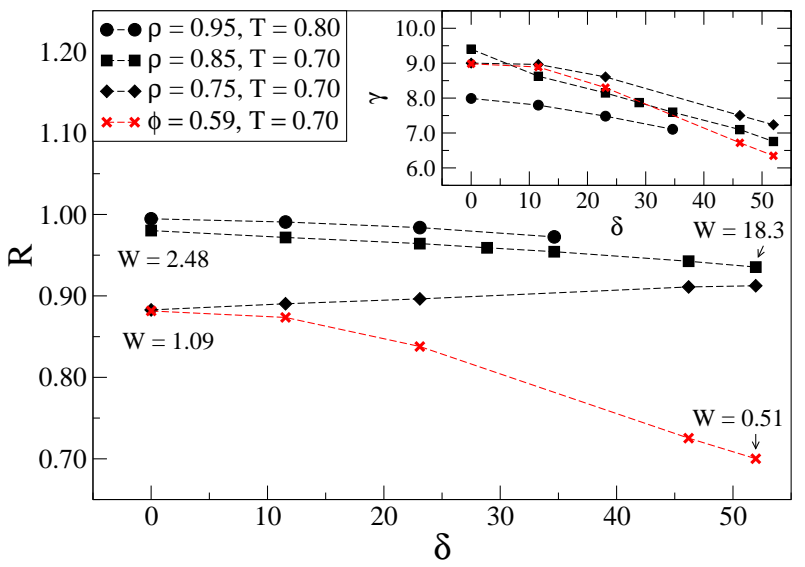

FIG. 4: Effect of polydispersity $\delta$ on the correlation coefficient $R$ (main figure, see Eq. (4)) and density-scaling exponent $\gamma$ $\equiv\langle\Delta U \Delta W\rangle /\left\langle(\Delta U)^{2}\right\rangle$ (inset) for the polydisperse LJ liquid in two dimensions. Black data points are for constant density $\rho$, and red data points are for constant volume (area) fraction $\phi$. For $0 \%$ polydispersity, $\rho=0.85$ is inside the coexistence region, and $\rho=0.95$ is inside the crystalline region.

responding volume fractions along the three simulated constant-density curves. For the density-scaling exponent $\gamma$, we only observe a decrease in its value, when polydispersity is increased.

\begin{tabular}{|l||l|l|l|}
\hline \hline$\delta$ & $\phi(\rho=0.75)$ & $\phi(\rho=0.85)$ & $\phi(\rho=0.95)$ \\
\hline \hline 0 & 0.589 & 0.668 & 0.746 \\
11.55 & 0.597 & 0.676 & 0.755 \\
23.09 & 0.619 & 0.702 & 0.784 \\
34.64 & - & 0.746 & 0.834 \\
51.96 & 0.746 & 0.845 & - \\
\hline \hline
\end{tabular}

TABLE I: Selected polydispersities $\delta$ and corresponding volume (area) fractions $\phi$ along the three simulated constantdensity curves.

As mentioned previously, the quantity $h(\rho) / T$ is invariant along an isomorph (see Sec. III). The function $h(\rho)$ for a LJ liquid in two dimensions is given by

$$
h(\tilde{\rho})=\left(\gamma_{*} / 3-1\right) \tilde{\rho}^{6}+\left(2-\gamma_{*} / 3\right) \tilde{\rho}^{3} .
$$

In the equation above: $\tilde{\rho} \equiv \rho / \rho_{*}, \rho_{*}$ is a chosen reference density, and $\gamma_{*}$ is the value of $\gamma=\langle\Delta U \Delta W\rangle /\left\langle(\Delta U)^{2}\right\rangle$ obtained from the equilibrium fluctuations at $\rho_{*}$ (see Refs. 65 and 82 for additional details). In this and the following section the reference state point is $\rho_{*}=1$ and $T_{*}=$ 1. We now proceed to study isomorphs of a moderately polydisperse LJ liquid. Isomorphs are generated by the procedure stated below 65,82 :

1. A starting state point is chosen.

2. Density is varied by some percentage (typically 10$20 \%$ but can be even a factor of two $\left.{ }^{82}\right)$. 
3. The temperature of the isomorphic state point is calculated via Eq. (9) keeping $h(\rho) / T$ constant.

4. A simulation is performed at the predicted state point and the procedure is repeated.

Figure 5(a) shows RDFs along an isomorph with $20 \%$ density increase $\left(\gamma_{*}=7.02\right.$ and starting state point $\rho=0.85$ and $T=0.70)$. For reference, Fig. 5 (b) shows an isotherm with the same density increase. A good invariance of structure is seen along the isomorph, whereas structure on the isotherm clearly shows a poorer scaling.
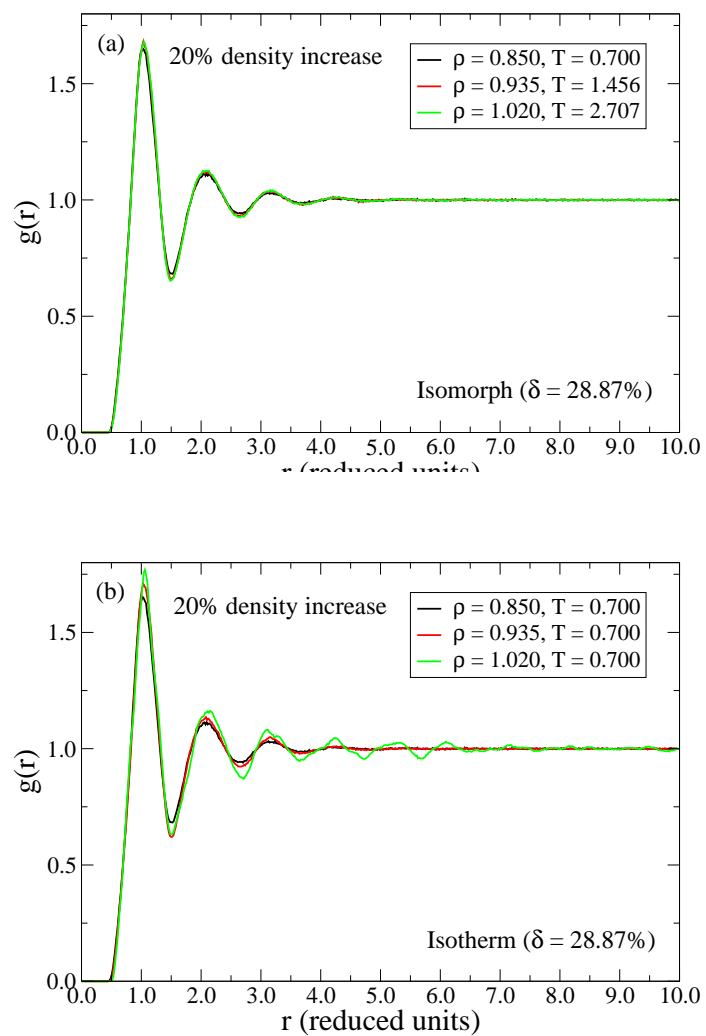

FIG. 5: RDFs for a moderately polydisperse LJ liquid ( $\delta$ $=28.87 \%$ ) in two dimensions. (a) Along an isomorph. (b) Along an isotherm. The highest density state point is not fully equilibrated.

Figure [6 shows the corresponding BOF figures. A good invariance is again seen along the isomorph but not along the isotherm.

Turning to the dynamics in Fig. 7] a near-perfect collapse is seen for the MSD on the isomorph whereas, as expected, a poor invariance is seen along the isotherm. The fact that liquids are highly size polydisperse is thus not a hindrance to showing simple isomorphic behavior.
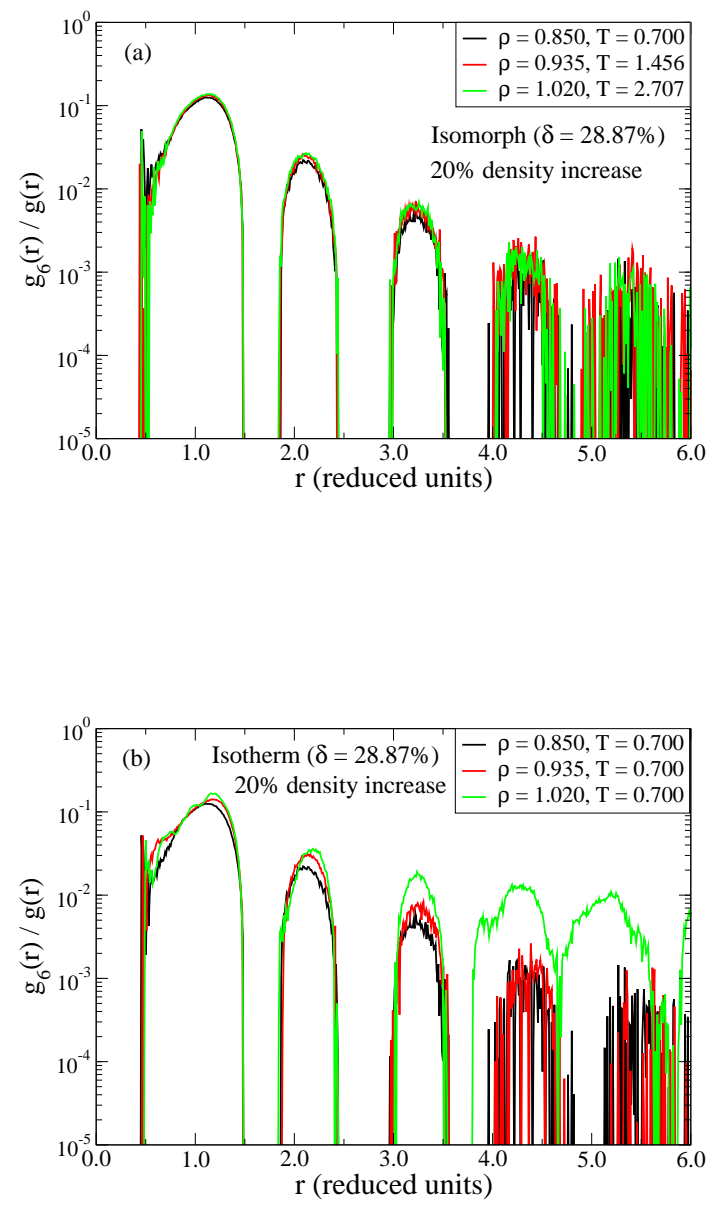

FIG. 6: BOFs for a moderately polydisperse LJ liquid ( $\delta$ $=28.87 \%$ ) in two dimensions. (a) Along an isomorph. (b) Along an isotherm. The highest density state point is not fully equilibrated.

\section{B. Simulations in three dimensions}

This section studies size polydisperse LJ liquids in three dimensions. Figure 8 shows the correlation coefficient $R$ as function of polydispersity at constant density and volume fraction. As for the two-dimensional results both a decrease and an increase in $R$ is observed, when polydispersity is increased at constant density (i.e., increasing pressure). For the density $\rho=0.75$, the liquid becomes RS at high polydispersities, whereas for $\rho$ $=0.85$ and $\rho=0.97$ all state points investigated are RS, albeit $R$ decreases. We observe again that highly size polydisperse LJ liquids are RS: a non-trivial observation. 

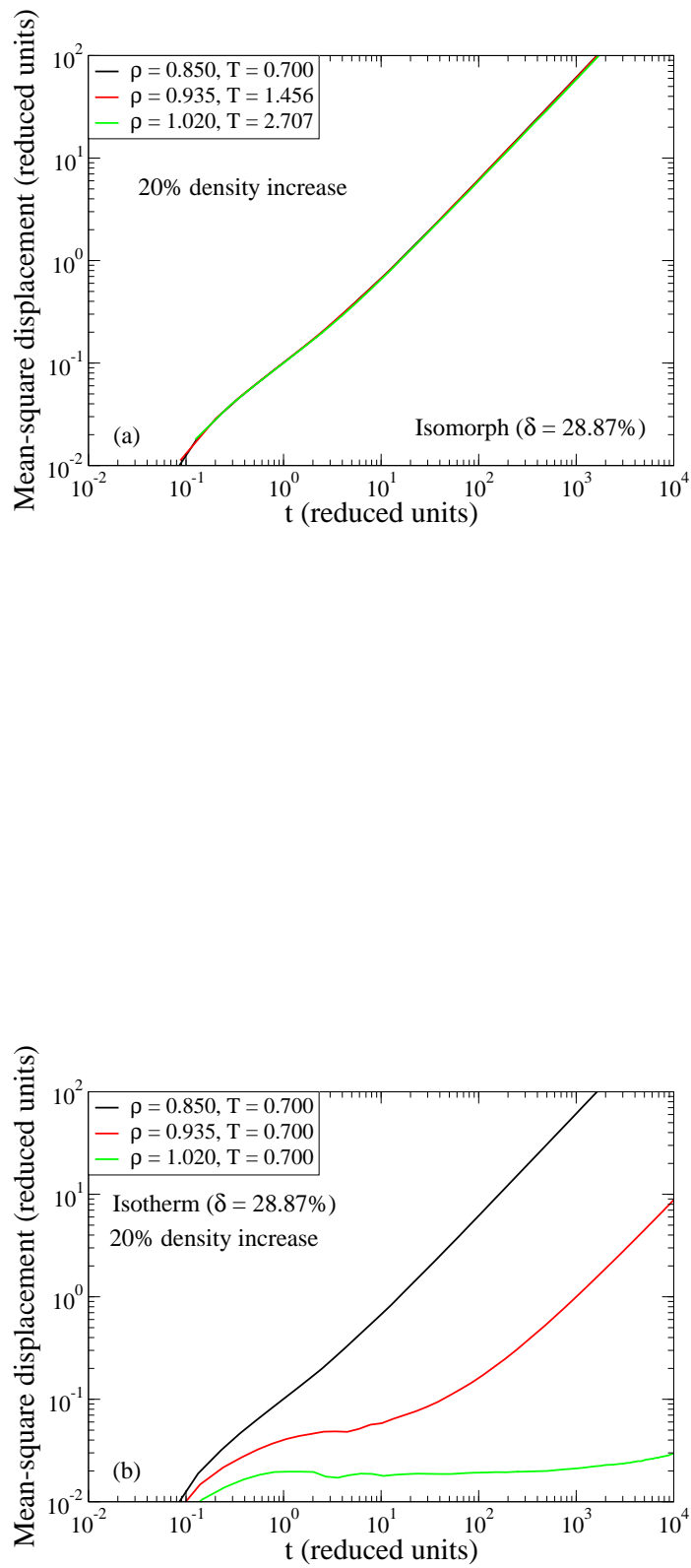

FIG. 7: MSDs for a moderately polydisperse LJ liquid ( $\delta$ $=28.87 \%$ ) in two dimensions. (a) Along an isomorph. (b) Along an isotherm. The highest density state point is not fully equilibrated.

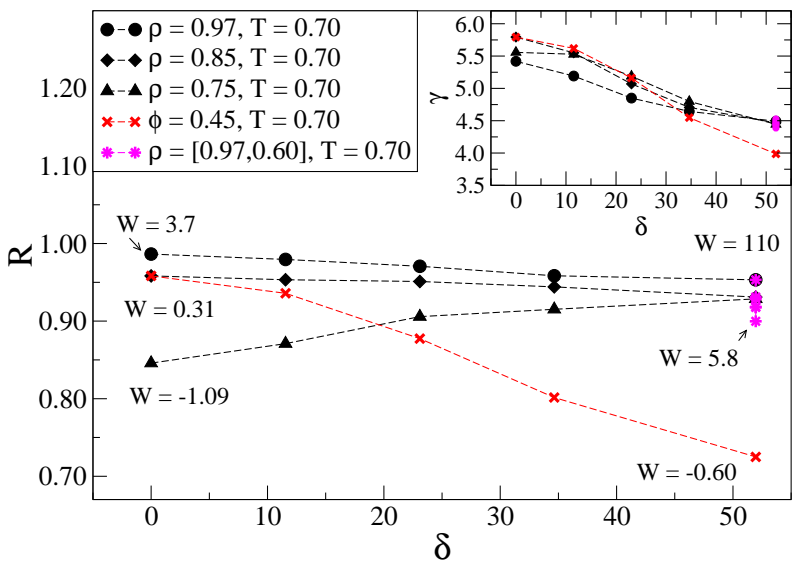

FIG. 8: Effect of polydispersity $\delta$ on the correlation coefficient $R$ (main figure, see Eq. (40) and density-scaling exponent $\gamma=$ $\langle\Delta U \Delta W\rangle /\left\langle(\Delta U)^{2}\right\rangle$ (inset) for the polydisperse LJ liquid in three dimensions. Black data points are for constant density $\rho$, and red data points are for constant volume fraction $\phi$. The highest polydispersity data points of $\rho=0.75$ and $\rho=$ 0.85, and the two highest polydispersity data points of $\rho=$ 0.97 are not fully equilibrated due to very slow dynamics. For 0\% polydispersity: $\rho=0.97$ is inside the crystalline region. The magenta asteriks data points span densities $(T=0.70)$ : $0.97,0.85,0.75,0.70,0.65$, and 0.60 .

In the case of constant volume fraction, a significant decrease in $R$ is observed and is accompanied by the virial turning negative (but not the pressure). Actually, the liquid goes from RS to non-RS in the studied range of polydispersities. For single-component liquids: $R$ usually decreases rapidly, when the virial becomes negative ${ }^{60}$.

The density-scaling exponent $\gamma$ shows - as in two dimensions - a decrease in its value as function of polydispersity. Actually, it is observed from Fig. 8 that all constant-density curves collapse at high polydispersities with $\gamma \approx 4.5$. Even a path at $\delta=52 \%$, where density varies from $\rho=0.97$ to 0.60 ( $\phi=0.94$ to 0.58 ) and the virial from $W=110$ to 5.8 , gives approximately the same value of $\gamma$ (see magenta asteriks data points).

This observation indicates that for highly size polydisperse LJ liquids $\gamma$ is mainly controlled by the degree of polydispersity $\delta$. In the non-reformulated isomorph theory $\gamma$ is predicted to be a function only of density 65 (see Sec. IIII). For the SCLJ liquid, a value of $\gamma \approx 5.5$ is obtained at low-to-moderate pressures (see inset and Ref. 65).

Very recently, an expression was established relating $\gamma$ to the pair potential of monatomic RS liquids 91 . The expression is given by

$$
\begin{aligned}
\gamma(\rho) & =\left.\frac{n^{(2)}(r)}{3}\right|_{r=\Lambda \rho^{-1 / 3}}, \\
n^{(2)}(r) & \equiv-2-r \frac{v^{(3)}(r)}{v^{(2)}(r)},
\end{aligned}
$$

where $\Lambda$ is a dimensionless number, and $v^{(p)}$ is the $p^{\prime}$ th 
derivative of the pair potential $v . \gamma$ is then related to the curvature and torsion of the pair potential. $\Lambda$ is chosen as the reduced distance $\left(\tilde{r}=r \rho^{1 / 3}\right)$ at which $r^{2} g(r)$ obtains its maximum, i.e., the most likely nearest-neighbor distance ${ }^{91}$. In this case, however, $\gamma$ is not a function only of density.

RDFs along an isomorph and isotherm for a polydisperse LJ liquid with $\delta=23.09 \%$ are given in Fig. $9\left(\gamma_{*}\right.$ $=4.79$ and starting state point $\rho=0.97$ and $T=0.70)$. The isomorph is generated via the aforementioned procedure keeping $h(\rho) / T$ constant. The expression 65 for $h(\rho)$ in three dimensions is given by

$$
h(\tilde{\rho})=\left(\gamma_{*} / 2-1\right) \tilde{\rho}^{4}+\left(2-\gamma_{*} / 2\right) \tilde{\rho}^{2},
$$

with definitions provided in the previous section. The $\mathrm{RDF}$ is to a good approximation invariant along the isomorph and less so on the isotherm (the highest density state point is, however, not fully equilibrated due to slow dynamics).
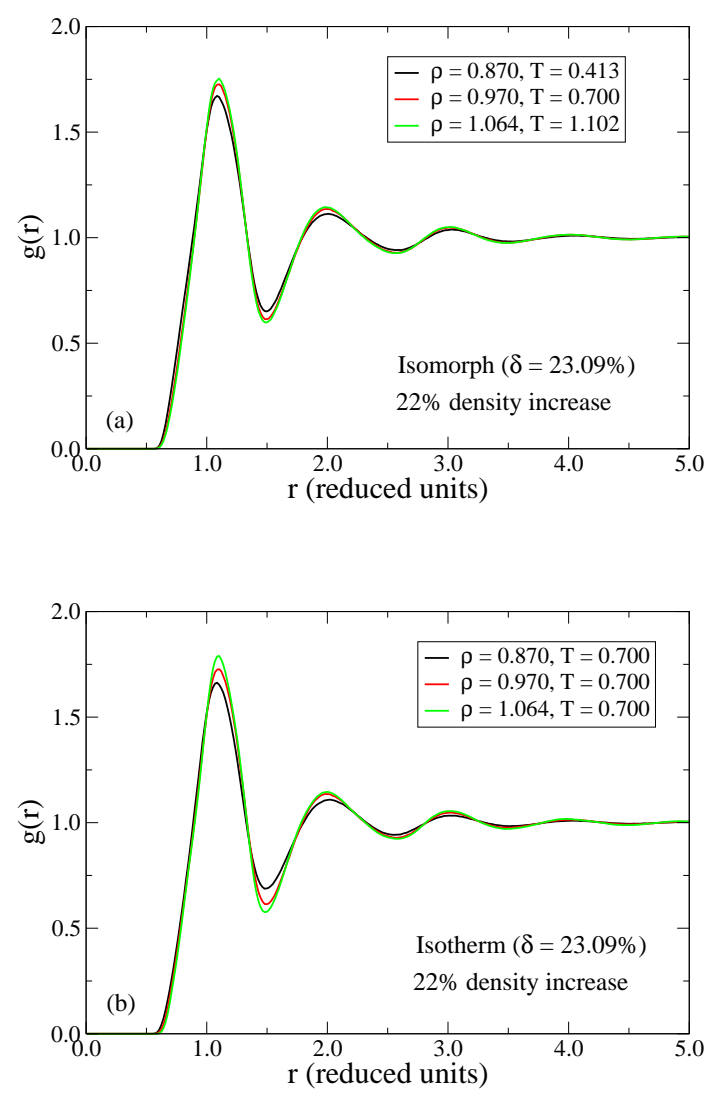

FIG. 9: RDFs for a moderately polydisperse LJ liquid $(\delta=$ $23.09 \%$ ) in three dimensions. (a) Along an isomorph. (b) Along an isotherm. The highest density state point is not fully equilibrated.

Figure 10] shows BOFs along the same isomorph and isotherm. The BOF is defined in three dimensions via

$$
g_{6}(r) \equiv \frac{L^{3}}{4 \pi r^{2} \Delta r N(N-1)} \sum_{i \neq k} \delta\left(r-\left|\mathbf{r}_{i k}\right|\right) S_{6}^{i k},
$$

in which

$$
\begin{aligned}
S_{6}^{i k} & \equiv \frac{4 \pi}{12+1} \sum_{m=-6}^{6} Q_{6 m}^{i} Q_{6 m}^{k *} \\
Q_{6 m}^{i} & \equiv \frac{1}{n_{i}+1} \sum_{g=1}^{n_{i}+1} \frac{1}{n_{g}} \sum_{j=1}^{n_{g}} Y_{6 m}\left(\mathbf{r}_{j g}\right) .
\end{aligned}
$$

The above expression for $Q_{6 m}^{i}$ gives coarse-grained Steinhardt bond-orientational-order parameters $90,92$. $Y_{6 m}$ is the spherical harmonic function with degree $l=$ 6 and order $m=[-6,6]$. The notation $n_{i}+1$ indicates that the sum is taken over all the nearest neighbors of particle $i$ including particle $i$ itself; nearest-neighbor particles are identified via the twelve-closest particles. From Fig. 10, a similar conclusion as for the RDF is reached; a good invariance is observed along the isomorph and less so on the isotherm. These results also indicate a good invariance of the first coordination shell (FCS) environment along the isomorph and are consistent with the role of the FCS in determining the structure and dynamics of RS liquids (see Ref. 66 for more information on FCS results).

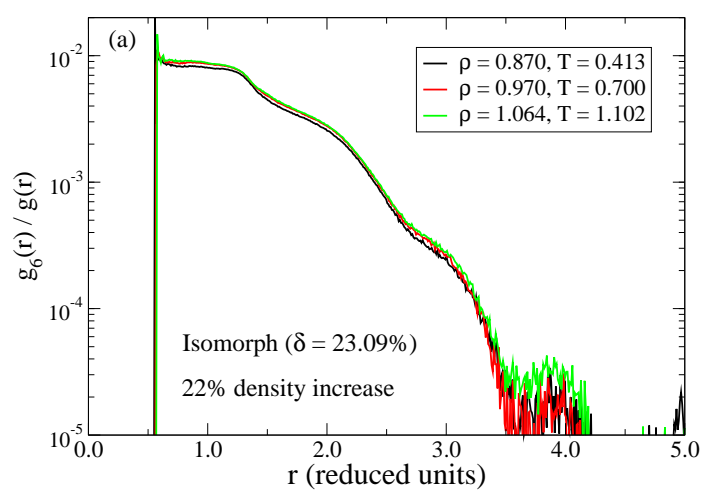




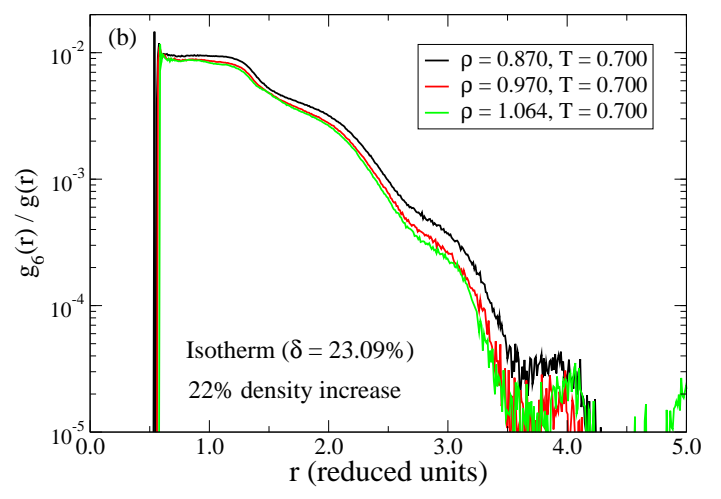

FIG. 10: BOFs for a moderately polydisperse LJ liquid ( $\delta$ $=23.09 \%$ ) in three dimensions. (a) Along an isomorph. (b) Along an isotherm. The highest density state point is not fully equilibrated.

Finally, the dynamics in terms of the MSD is shown in Fig. 11. An excellent invariance is obtained along the isomorph, whereas the isotherm shows approximately five orders-of-magnitude reduction in the diffusion coefficient.

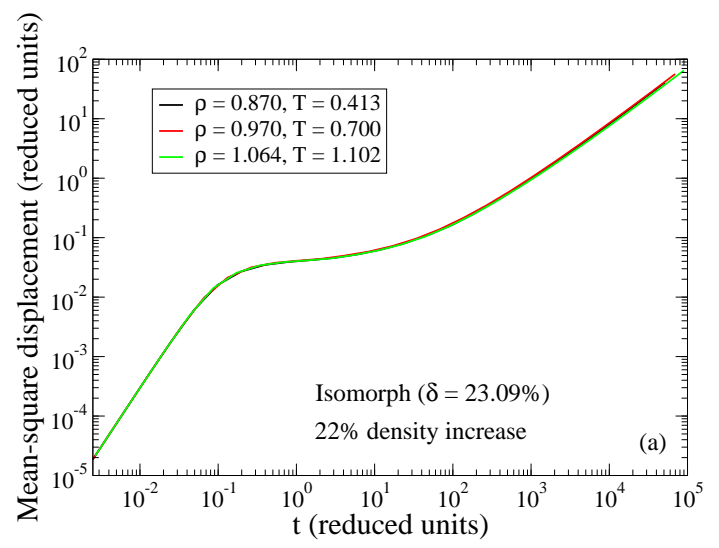

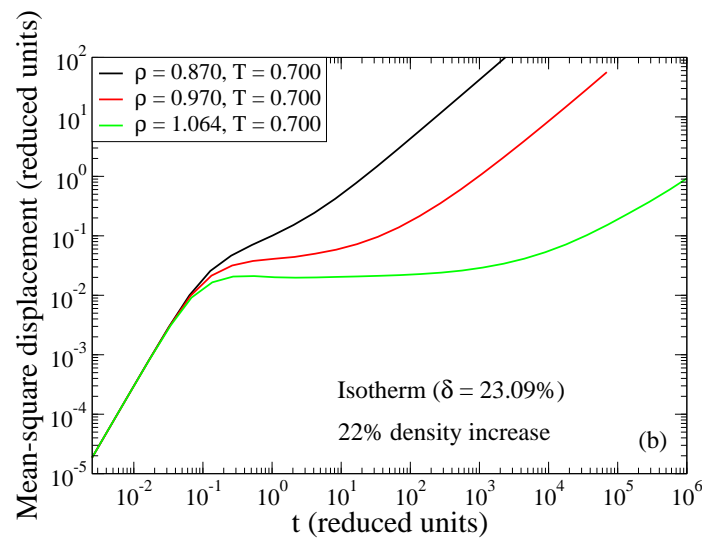

FIG. 11: MSDs for a moderately polydisperse LJ liquid ( $\delta$ $=23.09 \%$ ) in three dimensions. (a) Along an isomorph. (b) Along an isotherm. The highest density state point is not fully equilibrated.

\section{DISCUSSION}

We have demonstrated that both two- and threedimensional size polydisperse LJ liquids belong to the class of RS liquids up to a significant level of polydispersity. This shows that the theory of isomorphs can be applied to multi-component systems which contributes to the simpler physical description of this important class of liquids. Nevertheless, our simulations also showed unusual behavior when compared to the SCLJ liquid. 1) At higher densities, $R$ decreased with increasing polydispersity (pressure). An increase in pressure normally reduces interparticle distances in which a pure IPL description is a better approximation, and one would $a$ priori expect $R$ to increase. 2) For high polydispersities, the density-scaling exponent $\gamma$ is controlled only by the degree of polydispersity exhibiting also significantly lower $\gamma$-values at low-to-moderate pressures $(\gamma \approx 4.5$ versus $\gamma_{S C L J} \approx 5.5$ in three dimensions). Below we try to understand these observations in more detail presenting three-dimensional results only.

Figure 12]shows like-like RDFs (colored curves) for the small, medium, and large particles at $\rho=0.85, T=0.70$, $\delta=52 \%$, and the corresponding SCLJ liquid RDF (black curve). The RDFs for the medium and large particles move to shorter distances when compared to the SCLJ liquid (note the scaled distance axis). Conversely, the $\mathrm{RDF}$ for the small particles moves to larger distances and shows a very broad distribution. The higher value of the first peak is due to a clustering tendency of small particles in spaces surrounded by bigger particles (see Fig. 31)

The results signify a decoupling, with respect to pressure, amongst the small and large particles and could be the reason why $R$ decreases with increasing polydispersity (pressure) at higher densities. To verify this, we simulated at the same state point (not shown) a truncated uniform distribution excluding all the small(er) particles 
(i.e., particles with $\left.\sigma_{i}<1\right)$. In this case: $R$ increased with polydispersity (pressure).

Despite that the small particles are located around the minimum of the LJ potential, fluctuations in their positions are quite large. We speculate that this largeamplitude positional fluctuation might diminish the impact of the particle-size dependent inhomogeneous stress distribution on $R$ (see Eq. (5)). Accordingly, it may also explain why the liquid remains RS up to such high polydispersities. Additional studies are, however, needed to clarify this issue.

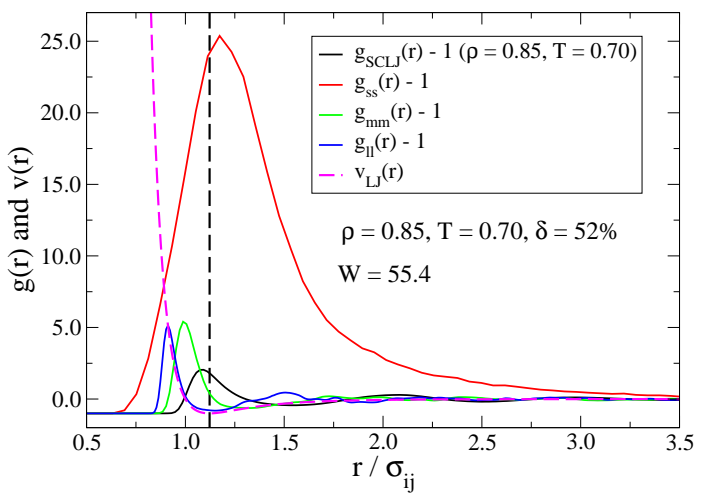

FIG. 12: Like-like RDFs for the small $(s)$, medium $(m)$, and large $(l)$ particles at $\rho=0.85, T=0.70, \delta=52 \%$ (colored curves; $W=55.4)$ in three dimensions. $g_{s s}(r)$ uses particle sizes in the range $\sigma_{i}=[0.1,0.2], g_{m m}(r)$ uses particle sizes in the range $\sigma_{i}=[1.0,1.1]$, and $g_{l l}(r)$ uses particle sizes in the range $\sigma_{i}=[1.8,1.9]$. We note that this state point is not fully equilibrated. The black curve gives SCLJ liquid RDF at $\rho=$ 0.85 and $T=0.70(W=0.31)$. The magenta dashed curve gives the LJ pair potential, and the black vertical dashed line delimits the minimum of the LJ potential.

The investigation is now focused on $\gamma$-values at low-tomoderate pressures for high polydispersities $(\gamma \approx 4.5)$. In the light of the theoretical results on monatomic RS liquids (see Eq. (10)), Fig. 13(a) shows $r^{2} g_{\alpha \alpha}(r)$ for the small $(s)$, medium $(m)$, and large $(l)$ particles $(\alpha=s, m$, l) at $\rho=0.60, T=0.70, \delta=52 \%(W=5.8)$. Figure 13(b) shows $n^{(2)}(r)$ for the LJ potential (Eq. (11)). Disregarding now the fact that the liquid is polydisperse we apply $n^{(2)}(r)$ to make predictions for $\gamma_{\alpha}$ using the procedure mentioned in Sec. IVB for RS monatomic liquids.

In this way $\gamma_{m}=5.5$ and $\gamma_{l}=4.9$ are obtained for the medium and large particles, respectively, whereas $\gamma_{s}=-2.9$ gives a negative value due to the divergence in $n^{(2)}(r)$. Interestingly, $\gamma_{m}$ gives a value very close to that expected for a SCLJ liquid at low-to-moderate pressures $\left(\gamma_{S C L J} \approx 5.5\right)$. Nevertheless, the results indicate that the large particles play an important role in determining the value of $\gamma$ for polydisperse LJ liquids with $\gamma_{l}=4.9$. It is, however, unclear which role the small particles play, as $r^{2} g_{s s}(r)$ peaks at very large distances with a resulting negative $\gamma_{s}$. We speculate that there is some cancellation between the medium particles of large $\gamma$ and the small particles of negative $\gamma$. More in-depth investigations are, however, needed.
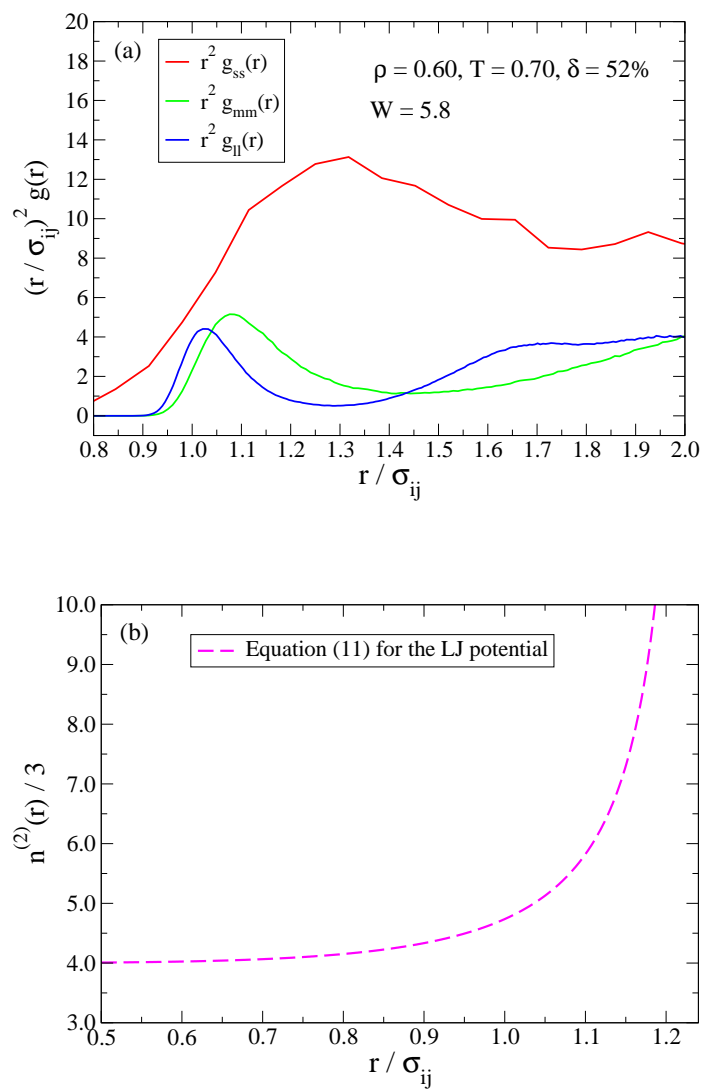

FIG. 13: (a) $r^{2} g_{\alpha \alpha}(r)$ for the small $(s)$, medium $(m)$, and large $(l)$ particles $(\alpha=s, m, l)$ at $\rho=0.60, T=0.70, \delta=$ $52 \%(W=5.8)$ in three dimensions. $g_{s s}(r)$ uses particle sizes in the range $\sigma_{i}=[0.1,0.2], g_{m m}(r)$ uses particle sizes in the range $\sigma_{i}=[1.0,1.1]$, and $g_{l l}(r)$ uses particle sizes in the range $\sigma_{i}=[1.8,1.9]$. (b) $n^{(2)}(r)$ of Eq. (11) for the LJ potential.

\section{CONCLUSION AND OUTLOOK}

Polydisperse fluids show rich phenomenology with respect to their single-component fluid counterparts and manifest themselves in as diverse systems as bitumen in road construction ${ }^{93}$ to micelles in biological systems ${ }^{41}$. In spite of this fact, we have shown that even highly size polydisperse LJ liquids belong to the class of RS liquids. As a consequence, polydisperse LJ liquids inherit the associated simple properties of RS liquids ${ }^{63,66}$ and are exemplified here by studying isomorphs of moderately polydisperse LJ liquids in both two and three dimensions.

The SCLJ liquid is a prime example of a RS liquid, however, many more model and experimental liquids are identified to belong to this class of liquids. A natural question thus arises: How are the remaining RS liquids 
affected by introducing size polydispersity? As an example, identical chain length LJ-bead polymer models have very recently been identified as $\mathrm{RS}^{76}$. For polymers the relevant size polydispersity is given by a distribution of chain lengths (mass distribution); can one expect such polymeric liquids to be RS? The answer is most likely in the affirmative. It is known from experiments that scaling relations typical of RS liquids, such as density scaling and excess entropy scaling, are also valid for polymers 83 . As another example, rigid-bond molecular liquids with LJ interactions have also been identified as RS94; it is expected that these liquids remain RS when introducing size polydispersity. A more general hypothesis is that all RS liquids will remain so to a high degree of accuracy when introducing size polydispersity. Future research should, however, focus on clarifying to what extent this hypothesis is valid and on understanding the implications thereof.

To conclude, the theory of isomorphs extends readily to multi-component systems, and when doing so it can be applied to improve even further the understanding of these intriguing systems. Experimental liquids are often distributed in additional or other variables such as mass or charge $\frac{7,18,41}{}$. More work in this direction is also needed to understand how these factors influence the results presented here.

\section{Acknowledgments}

Valuable discussions with Lorentzo Costigliola, Jeppe C. Dyre, Claire Lemarchand, and Thomas B. Schrøder are gratefully acknowledged. This work was partially supported by Grant-in-Aid for Scientific Research (S) and Specially Promoted Research from the Japan Society for the Promotion of Science (JSPS). T.S.I. acknowledges support from a JSPS Postdoctoral Fellowship. 
* Electronic address: trond@iis.u-tokyo.ac.jp

† Electronic address: tanaka@iis.u-tokyo.ac.jp

1 Bagchi, B. Molecular Relaxation in Liquids; Oxford University Press: New York, 2012.

2 Wolynes, P. G., Lubchenko, V., Eds. Structural Glasses and Supercooled Liquids: Theory, Experiment, and Applications; John Wiley and Sons, Inc., 2012.

3 Dickinson, E. Chem. Phys. Lett. 1978, 5\%, 148.

${ }^{4}$ Blum, L.; Stell, G. J. Chem. Phys. 1979, 71, 42.

5 Salacuse, J. J.; Stell, G. J. Chem. Phys. 1982, 77, 3714.

${ }^{6}$ Gualtieri, J. A.; Kincaid, J. M.; Morrison, G. J. Chem. Phys. 1982, 77, 521.

7 Ginoza, M.; Yasutomi, M. Mol. Phys. 1997, 91, 59.

8 Evans, R. M. L. Phys. Rev. E 1999, 59, 3192.

9 Sollich, P. J. Phys.: Condens. Matter 2002, $14, \mathrm{R} 79$.

10 Fasolo, M.; Sollich, P. Phys. Rev. Lett. 2003, 91, 068301.

11 Jacobs, W. M.; Frenkel, D. J. Chem. Phys. 2013, 139, 024108

12 Frenkel, D.; Vos, R. J.; de Kruif, C. G.; Vrij, A. J. Chem. Phys. 1986, 84, 4625.

13 Kofke, D. A.; Glandt, E. D. FFE 1986, 29, 327.

14 Kofke, D. A.; Glandt, E. D. J. Chem. Phys. 1987, 87, 4881.

15 Stapleton, M. R.; Tildesley, D. J.; Quirke, N. J. Chem. Phys. 1990, 92, 4456.

16 Auer, S.; Frenkel, D. Nature 2001, 413, 711.

17 Kristóf, T.; Liszi, J. Mol. Phys. 2001, 99, 167.

18 Murarka, R. K.; Bagchi, B. Phys. Rev. E 2003, 67, 051504.

19 Wilding, N. B.; Sollich, P. J. Phys.: Condens. Matter 2005, 17, S3245.

20 Wilding, N. B.; Sollich, P.; Fasolo, M. Phys. Rev. Lett. 2005, 95, 155701.

21 Wilding, N. B.; Sollich, P.; Fasolo, M.; Buzzacchi, M. J. Chem. Phys. 2006, 125, 014908.

22 Kawasaki, T.; Araki, T.; Tanaka, H. Phys. Rev. Lett. 2007, 99, 215701.

23 Abraham, S. E.; Bhattacharrya, S. M.; Bagchi, B. Phys. Rev. Lett. 2008, 100, 167801.

24 W.-S. Xu, Z.-Y. S.; An, L.-J. Phys. Rev. E 2012, 86, 041506

25 Sarkar, S.; Biswas, R.; Santra, M.; Bagchi, B. Phys. Rev. E 2013, 88, 022104.

${ }^{26}$ Ogarko, V.; Luding, S. Soft Matter 2013, 9, 9530.

27 Williamson, J. J.; Evans, R. M. L. Soft Matter 2013, 9, 3600.

28 Ashton, D. J.; Jack, R. L.; Wilding, N. B. Soft Matter 2013, 9, 9661.

29 Nguyen, D.-H.; Azéma, E.; Radjai, F.; Sornay, P. Phys. Rev. E 2014, 90, 012202.

30 Phillips, C. L.; Glotzer, S. C. J. Chem. Phys. 2014, 137, 104901.

31 Sarkar, S.; Biswas, R.; Ray, P. P.; Bagchi, B. arXiv 2014, 1402.6879

32 Koningsveld, R.; Kleintjens, L. A. Macromolecules 1971, 4, 637 .

33 Cowell, C.; Vincent, B. J. Colloid Interface Sci. 1982, 87, 518.

${ }^{34}$ Weeks, E. R.; Crocker, J. C.; Levitt, A. C.; Schofield, A.; Weitz, D. A. Science 2000, 28\%, 627.

35 Ye, X.; Sridhar, T. Macromolecules 2005, 38, 3442.

36 Watanabe, K.; Tanaka, H. Phys. Rev. Lett. 2008, 100,
158002

37 Ballesta, P.; Duri, A.; Cipelletti, L. Nat. Phys. 2008, 4, 550.

38 Banerjee, S.; Ghosh, R.; Bagchi, B. J. Phys. Chem. B 2012, 116, 3713.

39 Sacanna, S.; Korpics, M.; Rodriguez, K.; ColónMeléndez, L.; Kim, S.-H.; Pine, D. J.; Yi, G.-R. Nat. Commun. 2013, 4, 1688.

40 Palberg, T. J. Phys.: Condens. Matter 2014, 26, 333101.

41 Stapleton, M. R.; Tildesley, D. J.; Sluckin, T. J.; N, Q. J. Phys. Chem. 1988, 92, 4788.

42 Tanaka, H. Eur. Phys. J. E 2012, 35, 113.

43 Tanaka, H.; Kawasaki, T.; Shintani, H.; Watanabe, K. Nat. Mater. 2010, 9, 324.

44 Abraham, S. E.; Bagchi, B. Phys. Rev. E 2008, 78, 051501.

45 Rosenfeld, Y. Phys. Rev. A 1977, 15, 2545.

46 Rosenfeld, Y. J. Phys.: Condens. Matter 1999, 11, 5415.

47 Dzugutov, M. Nature 1996, 381, 137.

48 Pond, M. J.; Errington, J. R.; Truskett, T. M. J. Chem. Phys. 2011, 135, 124513.

49 Mittal, J.; Errington, J. R.; Truskett, T. M. Phys. Rev. Lett. 2006, 96, 177804.

50 Mittal, J.; Errington, J. R.; Truskett, T. M. J. Phys. Chem. $B$ 2007, 111, 10054 .

51 Watanabe, K.; Kawasaki, T.; Tanaka, H. Nat. Mater. 2011, 10, 512 .

52 Ingebrigtsen, T. S.; Errington, J. R.; Truskett, T. M.; Dyre, J. C. Phys. Rev. Lett. 2013, 111, 235901.

${ }^{53}$ Hanakata, P. Z.; Douglas, J. F.; Starr, F. W. Nat. Commun. 2014, 5, 4163.

54 Dyre, J. C. Phys. Rev. E 2013, 87, 022106.

55 Ingebrigtsen, T. S.; Toxvaerd, S.; Heilmann, O. J.; Schrøder, T. B.; Dyre, J. C. J. Chem. Phys. 2011, 135, 104101.

56 Ingebrigtsen, T. S.; Toxvaerd, S.; Schrøder, T. B.; Dyre, J. C. J. Chem. Phys. 2011, 135, 104102.

57 Ingebrigtsen, T. S.; Dyre, J. C. J. Chem. Phys. 2012, 137, 244101.

${ }^{58}$ Bacher, A. K.; Dyre, J. C. Colloid. Polym. Sci. 2014, 292, 1971.

59 Bacher, A. K.; Schrøder, T. B.; Dyre, J. C. Nat. Commun. 2014, 5, 5424.

60 Bailey, N. P.; Pedersen, U. R.; Gnan, N.; Schrøder, T. B.; Dyre, J. C. J. Chem. Phys. 2008, 129, 184507.

61 Bailey, N. P.; Pedersen, U. R.; Gnan, N.; Schrøder, T. B.; Dyre, J. C. J. Chem. Phys. 2008, 129, 184508.

62 Schrøder, T. B.; Bailey, N. P.; Pedersen, U. R.; Gnan, N.; Dyre, J. C. J. Chem. Phys. 2009, 131, 234503.

63 Gnan, N.; Schrøder, T. B.; Pedersen, U. R.; Bailey, N. P.; Dyre, J. C. J. Chem. Phys. 2009, 131, 234504.

64 Schrøder, T. B.; Gnan, N.; Pedersen, U. R.; Bailey, N. P.; Dyre, J. C. J. Chem. Phys. 2011, 134, 164505.

65 Ingebrigtsen, T. S.; Bøhling, L.; Schrøder, T. B.; Dyre, J. C. J. Chem. Phys. 2012, 136, 061102.

66 Ingebrigtsen, T. S.; Schrøder, T. B.; Dyre, J. C. Phys. Rev. $X$ 2012, 2, 011011.

67 Gundermann, D.; Pedersen, U. R.; Hecksher, T.; Bailey, N. P.; Jakobsen, B.; Christensen, T.; Olsen, N. B.; Schrøder, T. B.; Fragiadakis, D.; Casalini, R.; Roland, C. M.; Dyre, J. C.; Niss, K. Nat. Phys. 2011, 7, 816. 
68 Roed, L. A.; Gundermann, D.; Dyre, J. C.; Niss, K. J. Chem. Phys. 2013, 139, 101101.

69 Xiao, W.; Tofteskov, J.; Christensen, T. V.; Dyre, J. C.; Niss, K. J. Non-Crystal. Solids 2014, http://dx.doi.org/10.1016/j.jnoncrysol.2014.08.041

70 Separdar, L.; Bailey, N. P.; Schrøder, T. B.; Davatolhagh, S.; Dyre, J. C. J. Chem. Phys. 2013, 138, 154505.

71 Nosé, S. J. Chem. Phys. 1984, 81, 511.

72 Hoover, W. G. Phys. Rev. A 1985, 31, 1695.

73 Toxvaerd, S. Mol. Phys. 1991, 72, 159.

74 Wilding, N. B.; Sollich, P. J. Chem. Phys. 2010, 133, 224102.

75 Allen, M. P.; Tildesley, D. J. Computer Simulation of Liquids; Oxford University Press: New York, 1987.

76 Veldhorst, A. A.; Dyre, J. C.; Schrøder, T. B. J. Chem. Phys 2014, 141, 054904.

77 Coslovich, D.; Roland, C. M. J. Phys. Chem. B 2008, 112, 1329.

78 Coslovich, D.; Roland, C. M. J. Chem. Phys. 2009, 130, 014508 .

79 Coslovich, D.; Roland, C. M. J. Chem. Phys. 2009, 131, 151103.

80 Dyre, J. C. J. Phys. Chem. B 2014, 118, 10007.

81 Hoover, W. G.; Ross, M.; Johnson, K. W.; Henderson, D.; Barker, J. A.; Brown, B. C. J. Chem. Phys. 1970, 52, 4931.

82 Bøhling, L.; Ingebrigtsen, T. S.; Grzybowski, A.;
Paluch, M.; Dyre, J. C.; Schrøder, T. B. New J. Phys. 2012, 14, 113035.

83 Roland, C. M. Macromolecules 2010, 43, 7875.

84 Ingebrigtsen, T. S.; Dyre, J. C. Soft Matter 2014, 10, 4324.

85 Albrechtsen, D. E.; Olsen, A. E.; Pedersen, U. R.; Schrøder, T. B.; Dyre, J. C. Phys. Rev. B 2014, 90, 094106.

86 Rosenfeld, Y.; Tarazona, P. Mol. Phys. 1998, 95, 141.

87 Ingebrigtsen, T. S.; Veldhorst, A. A.; Schrøder, T. B.; Dyre, J. C. J. Chem. Phys. 2013, 139, 171101.

88 Schrøder, T. B.; Dyre, J. C. J. Chem. Phys. 2014, 141, 204502.

89 Binder, K.; Sengupta, S.; Nielaba, P. J. Phys.: Condens. Matter 2002, 14, 2323.

90 Steinhardt, P. J.; Nelson, D. R.; Ronchetti, M. Phys. Rev. $B$ 1983, 28, 784 .

91 Bøhling, L.; Bailey, N. P.; Schrøder, T. B.; Dyre, J. C. J. Chem. Phys. 2014, 140, 124510.

92 Lechner, W.; Dellago, C. J. Chem. Phys. 2008, 129, 114707.

93 Hansen, J. S.; Lemarchand, C. A.; Nielsen, E.; Dyre, J. C.; Schrøder, T. J. Chem. Phys. 2013, 138, 094508.

94 Ingebrigtsen, T. S.; Schrøder, T. B.; Dyre, J. C. J. Phys. Chem. B 2012, 116, 1018. 